\title{
ON AN INVERSE TO THE HÖLDER INEQUALITY
}

\section{J. PEČARIĆ}

Faculty of Textile Technology

Zagreb University

Zagreb

\section{C.E.M. PEARCE}

Applied Mathematics Department University of Adelaide

Adelaide 5005

South Australia

(Received October 5, 1994)

ABSTRACT. An extension is given for the inverse to Hölder's inequality obtained recently by Zhuang.

KEY WORDS AND PHRASES. Inverse Hölder inequality.

1991 AMS SUBJECT CLASSIFICATION CODE. 26D15.

Recently Zhuang [1] proved the following inverse of the arithmetico-geometric inequality.

THEOREM A. Let $0<a \leq x \leq A, 0<b \leq y \leq B, 1 / p+1 / q=1, p>1$; then

$$
\frac{x}{p}+\frac{y}{q} \leq \max \left\{\frac{A / p+b / q}{A^{1 / p} b^{1 / q}}, \frac{a / p+B / q}{a^{1 / p} B^{1 / q}}\right\} x^{1 / p} y^{1 / q},
$$

or

$$
x+y \leq \max \left\{\frac{A+b}{A^{1 / p} b^{1 / q}}, \frac{a+B}{a^{1 / p} B^{1 / q}}\right\} x^{1 / p} y^{1 / q},
$$

the sign of equality in (1) and (2) holds if and only if either $(x, y)=(a, B)$ or $(x, y)=(A, b)$. Moreover, if $a \geq B$, then

$$
\frac{a / p+B / q}{a^{1 / p} B^{1 / q}} x^{1 / p} y^{1 / q} \leq \frac{x}{p}+\frac{y}{q} \leq \frac{A / p+b / q}{A^{1 / p} b^{1 / q}} x^{1 / p} y^{1 / q}
$$

the sign of equality on the right-hand side of (3) holds if and only if $(x, y)=(A, b)$, and the sign of equality on the left-hand side of $(3)$ holds if and only if $(x, y)=(a, B)$. The sign of inequality in (3) is reversed if $b \geq A$.

This enables us to formulate the following theorem.

THEOREM 1. Suppose $x, y, a, b, A, B, p, q$ are as in Theorem $\mathrm{A}$ and $\alpha, \beta>0$. Then

$$
\alpha x^{p}+\beta y^{q} \leq \max (C, D) x y
$$

where

$$
C=\left(\alpha A^{p}+\beta b^{q}\right) /(A b), \quad D=\left(\alpha a^{p}+\beta B^{q}\right) /(a B) .
$$

Equality occurs if and only if either $(x, y)=(a, B)$ or $(x, y)=(A, b)$. Moreover, if $\alpha p a^{p} \geq$ $\beta q B^{q}$, then

$$
C x y \leq \alpha x^{p}+\beta y^{q} \leq D x y,
$$

with equality on the right-hand side if and only if $(x, y)=(A, b)$ and on the left if and only if $(x, y)=(a, B)$. The inequalities in (6) are reversed if $\alpha p A^{p} \leq \beta q b^{q}$. 
PROOF. Inequalities (4) and (6) follow from (1) and (3) under the substitutions

$$
x \rightarrow \alpha p x^{p}, y \rightarrow \beta q y^{q}, a \rightarrow \alpha p a^{b}, \quad b \rightarrow \beta q b^{q}, \quad A \rightarrow \alpha p A^{p} . \quad B \rightarrow \beta q B^{q} .
$$

REMARK. Theorem 1 gives (1) and (2) together, (1) resulting from the substitutions $\alpha=1 / p$, $x \rightarrow x^{1 / p}, A=A^{1 / p}, a \rightarrow a^{1 / p}$ and corresponding relations for $\beta, y$ etc. with $q$ in place of $p$, while (2) results from similar substitutions with $\alpha=1=\beta$.

The following result now gives an extension of the inverse to Hölder's inequality obtained in [1]. We suppose that all the integrals involved exist.

THEOREM 2. Let the functions $f, g$ satisfy $0<a \leq f(x) \leq A, 0<b \leq g(x) \leq B$ for almost all $x \in X$ with respect to a measure $\mu$. Suppose $\alpha, \beta, p, q, C, D$ are as in Theorem 1 . Then

$$
\left(\int_{X} f^{p} d \mu\right)^{1 / p}\left(\int_{X} g^{q} d \mu\right)^{1 / q} \leq(\alpha \beta)^{-1 / p}(\beta q)^{-1 / q} \max (C, D) \int_{X} f g d \mu
$$

and equality holds if and only if

$$
\mu\left(E_{1} \cup F_{1}\right)=\mu(X)
$$

and

where

$$
\mu\left(E_{1}\right)=\frac{\left(\alpha p A^{p}-\beta q b^{q}\right) \mu(X)}{\alpha p\left(A^{p}-a^{p}\right)+\beta q\left(B^{q}-\beta^{q}\right)},
$$

$$
\begin{aligned}
& E_{1}=\{x \in X: f(x)=a, g(x)=B\}, \\
& F_{1}=\{x \in X: f(x)=A, g(x)=b\} .
\end{aligned}
$$

Moreover, if $\alpha p a^{p} \geq \beta q B^{q}$, then

$$
\left(\int_{X} f^{p} d \mu\right)^{1 / p}\left(\int_{X} g^{q} d \mu\right)^{1 / q} \leq(\alpha p)^{-1 / p}(\beta q)^{-1 / q} D \int_{X} f g d \mu
$$

with equality only if $(f, g)=(a, B)$ a.e. on $X$ and $\alpha p a^{p}=\beta q B^{q}$, and if $\alpha p A^{p} \leq \beta q b^{q}$, then

$$
\left(\int_{X} f^{p} d \mu\right)^{1 / p}\left(\int_{X} g^{q} d \mu\right)^{1 / q} \leq(\alpha p)^{-1 / p}(\beta q)^{-1 / q} C \int_{X} f g d \mu
$$

with equality only if $(f, g)=(A, b)$ a.e. on $X$ and $\alpha p A^{p}=\beta q b^{q}$.

PROOF. The first statement was proved in [1]. A simple proof of the remainder of the theorem was given for the case $\alpha=1 / p, \beta=1 / q$ in [2]. We give a similar simple proof for the general case.

$$
\begin{aligned}
\max (C, D) \int_{X} f g d \mu & =\int_{X} \max (C, D) f g d \mu \\
& \geq \int_{X}\left(\alpha f^{p}+\beta g^{q}\right) d \mu \\
& =\frac{1}{p}(\alpha p) \int_{X} f^{p} d \mu+\frac{1}{q}(\beta q) \int_{X} g^{q} d \mu \\
& \geq(\alpha p)^{1 / p}(p q)^{1 / q}\left(\int_{X} f^{p} d \mu\right)^{1 / p}\left(\int_{X} g^{q} d \mu\right)^{1 / q}
\end{aligned}
$$

by the arithmetico-geometric inequality. 
The equality conditions result from those in Theorem 1 and the arithmetico-geometric inequality.

Similarly we can prove (8). Using the second inequality in (6) we have

$$
\begin{aligned}
D \int_{X} f g d \mu & =\int_{X} D f g d \mu \\
& \geq \int_{X}\left(\alpha f^{p}+\beta g^{q}\right) d \mu \\
& =\frac{1}{p}(\alpha p) \int_{X} f^{p} d \mu+\frac{1}{q}(\beta q) \int_{X} g^{q} d \mu \\
& \geq(\alpha p)^{1 / p}(p q)^{1 / q}\left(\int_{X} f^{p} d \mu\right)^{1 / p}\left(\int_{X} g^{q} d \mu\right)^{1 / q} .
\end{aligned}
$$

Relation (9) follows similarly.

REMARK. The simplest cases of (8) and (9) occur for $\alpha=1 / p, \beta=1 / q$. Then we have that if $a^{p} \geq B^{q}$, then

$$
\left(\int_{X} f^{p} d \mu\right)^{1 / p}\left(\int_{X} g^{q} d \mu\right)^{1 / q} \leq D_{1} \int_{X} f g d \mu
$$

and if $A^{p} \leq b^{q}$, then

$$
\left(\int_{X} f^{p} d \mu\right)^{1 / p}\left(\int_{X} g^{q} d \mu\right)^{1 / q} \leq C_{1} \int_{X} f g d \mu
$$

where

$$
\begin{aligned}
& D_{1}=\left(\frac{1}{p} a^{p}+\frac{1}{q} B^{q}\right) /(a B), \\
& C_{1}=\left(\frac{1}{p} A^{p}+\frac{1}{q} b^{q}\right) /(A b) .
\end{aligned}
$$

\section{REFERENCES}

1. ZHUANG, YA-DONG. On inverses of the Hölder inequality, J. Math. Anal. Applic., $\underline{161}$ (1991), 566-575.

2. MOND, B. and PECARIĆ, J.E. Remark on a recent converse of Hölder's inequality, J. Math. Anal. Applic., 181 (1994), 280-281. 


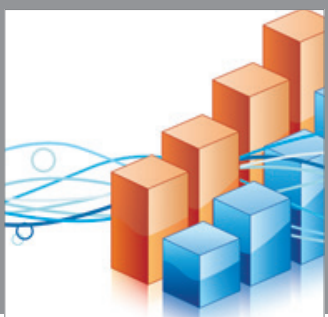

Advances in

Operations Research

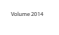

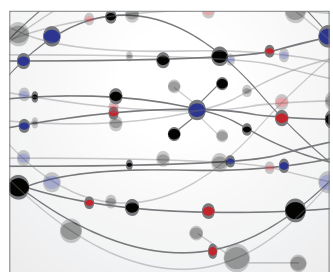

\section{The Scientific} World Journal
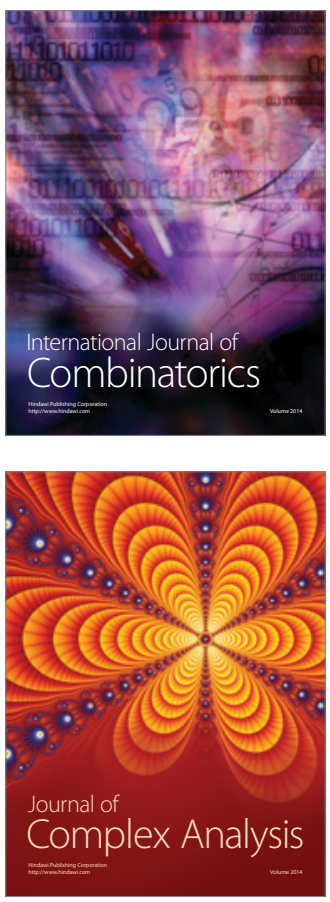

International Journal of

Mathematics and

Mathematical

Sciences
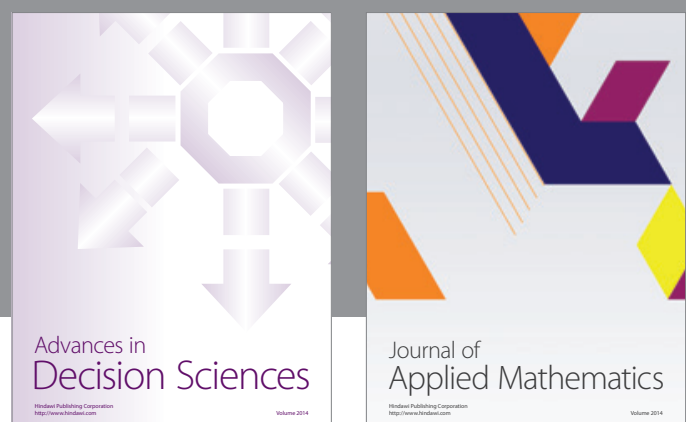

Journal of

Applied Mathematics
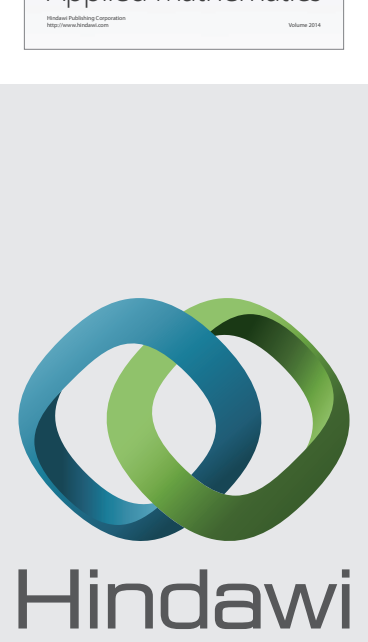

Submit your manuscripts at http://www.hindawi.com
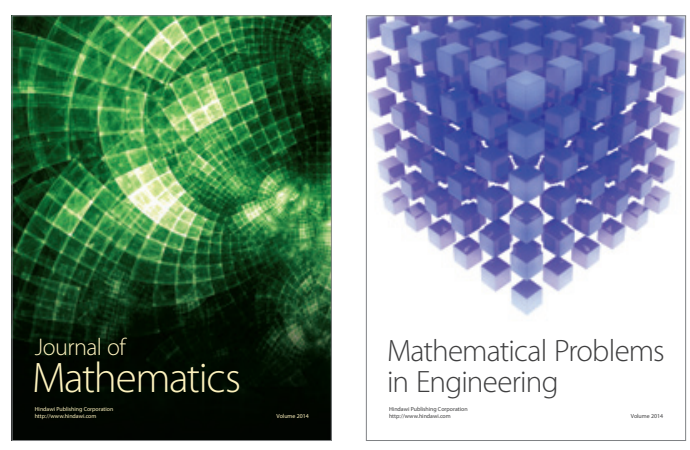

Mathematical Problems in Engineering
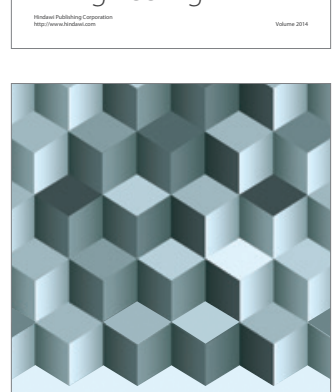

Journal of

Function Spaces
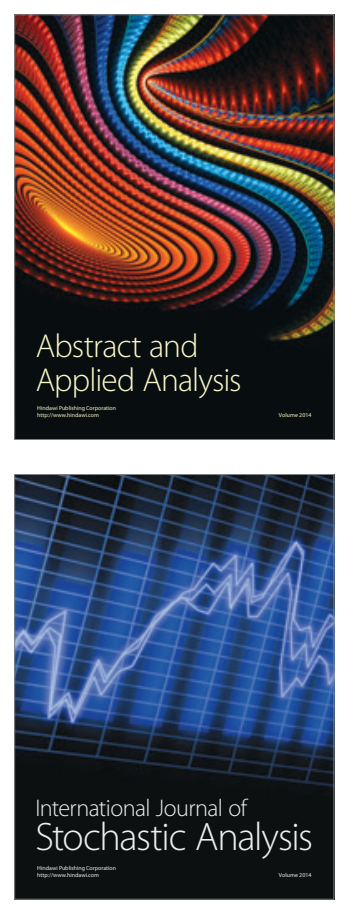



ournal of

Probability and Statistics

Promensencen
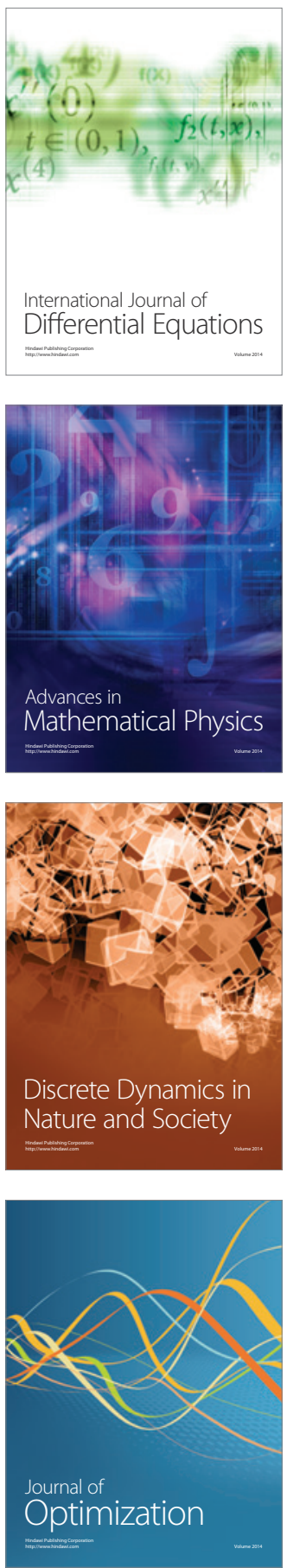